University of Nebraska - Lincoln

DigitalCommons@University of Nebraska - Lincoln

Timothy J. Gay Publications

Research Papers in Physics and Astronomy

2016

\title{
Method for monitoring GaAs photocathode heat cleaning temperature
}

\author{
Nathan B. Clayburn \\ University of Nebraska - Lincoln, nclayburn2@unl.edu \\ Kenneth Wayne Trantham \\ University of Nebraska at Kearney, tranthamkw@unk.edu \\ M. Dunn \\ University of Nebraska-Lincoln \\ Timothy J. Gay \\ University of Nebraska - Lincoln, tgay1@unl.edu
}

Follow this and additional works at: https://digitalcommons.unl.edu/physicsgay

Part of the Atomic, Molecular and Optical Physics Commons, and the Other Physics Commons

Clayburn, Nathan B.; Trantham, Kenneth Wayne; Dunn, M.; and Gay, Timothy J., "Method for monitoring GaAs photocathode heat cleaning temperature" (2016). Timothy J. Gay Publications. 73.

https://digitalcommons.unl.edu/physicsgay/73

This Article is brought to you for free and open access by the Research Papers in Physics and Astronomy at DigitalCommons@University of Nebraska - Lincoln. It has been accepted for inclusion in Timothy J. Gay Publications by an authorized administrator of DigitalCommons@University of Nebraska - Lincoln. 


\title{
Method for monitoring GaAs photocathode heat cleaning temperature
}

\author{
N. B. Clayburn, ${ }^{1}$ K. W. Trantham, ${ }^{2}$ M. Dunn, ${ }^{1}$ and T. J. Gay ${ }^{1}$ \\ ${ }^{1}$ Jorgensen Hall, University of Nebraska, Lincoln, Nebraska 68588-0299, USA \\ ${ }^{2}$ Department of Physics and Astronomy, University of Nebraska, Kearney, Nebraska 68849, USA
}

(Received 15 August 2016; accepted 1 December 2016; published online 21 December 2016)

\begin{abstract}
Before a GaAs photocathode can be activated to achieve a negative electron affinity condition, the GaAs crystal must be cleaned. This is most commonly done by ohmic, radiative, or electron bombardment heating. We report a new technique to monitor the temperature of heated GaAs photocathodes by observation with a camera. The method is robust and yields the same temperatures for different GaAs samples heated using different methods in different mounting configurations. Published by AIP Publishing. [http://dx.doi.org/10.1063/1.4972255]
\end{abstract}

\section{INTRODUCTION}

\section{A. NEA GaAs polarized electron sources}

When circularly polarized light with near-bandgap energy illuminates a negative electron affinity (NEA) GaAs photocathode, spin-polarized electrons are emitted. ${ }^{1-3} \mathrm{GaAs}$ crystals with NEA are said to be "activated." In order to obtain an NEA surface with good quantum efficiency (QE), alternating layers of $\mathrm{Cs}$ and $\mathrm{O}_{2}$ or $\mathrm{NF}_{3}$ are applied to an atomically clean GaAs crystal. The cleaning procedure involves either evaporation of the surface by heating it to just below the "congruent evaporation temperature" of $657^{\circ} \mathrm{C}$ (above which the $\mathrm{Ga}$ and As atoms leave the surface in non-stoichiometric proportions) or the chemical removal of contaminants at lower temperatures, typically with the use of atomic hydrogen. ${ }^{4}$ In the former case, which is more commonly used and is relevant for this paper, heating is accomplished either by passing electrical current through the sample (ohmic heating), placing a hot filament near the crystal (radiative heating), or bombarding the crystal with energetic electrons. Base pressures below $2 \times 10^{-10}$ Torr are generally necessary for these procedures.

A crystal's NEA state is not permanent, even in an ultra-high vacuum environment, due to surface deposition of contaminants and from bombardment by back streaming ions produced by collisions of photoelectrons with residual gas molecules. ${ }^{2}$ These mechanisms make it necessary to reheat clean and re-activate the crystal periodically. The level and composition of the surface contamination for each photocathode are unique, due to differences in photocathode preparation and non-identical vacuum conditions. Owing to these differences, the exact details of the heat cleaning and activation processes can vary significantly between individual source apparatus in general, and for each photocathode in particular. Thus, a general protocol must be developed for obtaining NEA conditions which makes allowance for these differences. It can easily take many months to establish such protocols for a new polarized electron source, and several attempts at activation for each new photocathode. Techniques which make heat cleaning and activations easier, such as those outlined in this report, can be very helpful in establishing consistent and routine operation of a GaAs polarized electron source.
The heat cleaning process is especially important, as improper heating above the congruent evaporation temperature can irreversibly damage the GaAs photocathode. At higher temperatures, incipient local evaporation of arsenic occurs resulting in gallium droplet formation on the surface. ${ }^{5-7}$ This process (Fig. 1) gives the crystal a "frosted" appearance and results in a significant reduction in $\mathrm{QE}$, often rendering the photocathode effectively unusable. ${ }^{7}$ More advanced cleaning methods like those discussed in Ref. 4 can achieve better results at lower temperatures using specific procedures for multiple re-cleanings by atomic hydrogen.

\section{B. Heat-cleaning monitoring using conventional instrumentation}

Numerous methods and/or instruments are used to monitor the temperatures of photocathodes during activation, including thermocouples, ${ }^{2,3,7,12}$ visual observation of the crystal, ${ }^{2,7-12}$ pyrometers, ${ }^{7,13}$ or the observation of laser beams reflected off the photocathode surface. ${ }^{13}$ Monitoring the chamber pressure and the resistance change of the photocathode can also be useful tools for this purpose. ${ }^{2,9}$ Each of these has its shortcomings; the general consensus is that measurements of the temperature of GaAs during heat cleaning are inaccurate and difficult to reproduce.

Measurements with a nearby thermocouple junction give a temperature closer to that of the structure holding the thermocouple than the crystal itself. Moreover, the relationship between crystal temperature and structure temperature will change with each installation. Non-contact thermometers such as IR pyrometers are expensive and often unreliable, because the spectral emissivity of the GaAs crystal is a complicated quantity to characterize ${ }^{14}$ and is subject to factors such as polish and ad-layer content. Even environmental factors, such as stray light from heaters or reflections inside the vacuum chamber, can affect these optical measurements. The observation of laser light reflected from the surface can provide some information about the crystal. If the crystal becomes frosted, then the reflected laser beam from the surface becomes diffuse. ${ }^{13}$ This can be an effective tool for determining if the crystal has become frosted and can provide a reference point for the crystal heating, but is not a complete method for 


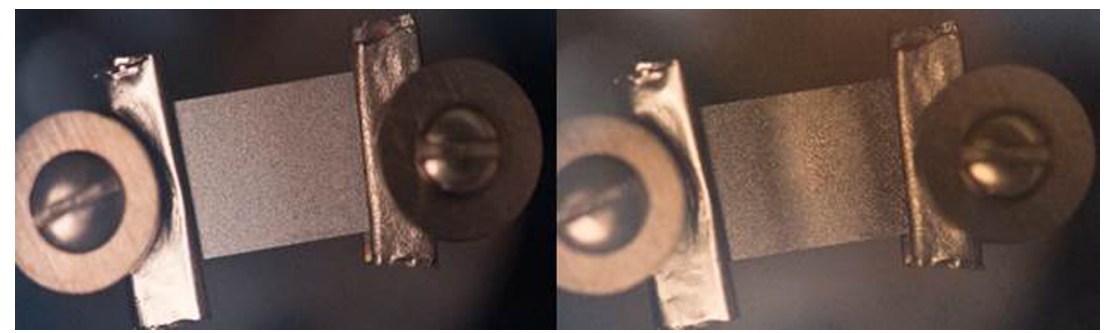

FIG. 1. A GaAs crystal mounted between two electrical terminals, with (left) no frosting, and (right) frosting produced by heating above $657^{\circ} \mathrm{C}$. The foil sandwiched between the terminal washers and the crystal are used to improve the uniformity of electrical contact for ohmic heating.

photocathode temperature monitoring. This method, and others that rely on the observation of the "frosted" condition, do not warn the user before the crystal is already irreversibly damaged and rendered unusable as a photocathode. Measuring resistance changes by monitoring the current and the voltage delivered to the crystal during ohmic heating can be useful too, but resistance is a property that is dependent on the photocathode size and the dopant density. Thus, it is hard to standardize such a technique.

\section{Visual heat-cleaning monitoring}

Because of the shortcomings of the methods outlined above, more qualitative visual methods are often used. With practice, simple observation of the GaAs crystal color can actually provide an effective means of qualitatively monitoring its temperature. ${ }^{12}$ When the crystal is heated between $~ 590$ and $610^{\circ} \mathrm{C}$, it just begins to emit visible light. At temperatures between $\sim 610$ and $650{ }^{\circ} \mathrm{C}$, a faint red glow becomes visible. At temperatures greater than $650{ }^{\circ} \mathrm{C}$, the crystal glows an intense red color. The process of learning to gauge the photocathode color and intensity at a temperature just below that which causes non-stoichiometric evaporation requires numerous heating attempts and can result in many ruined crystals. Because reaching sufficiently low pressures for heat cleaning generally requires baking the chamber, this can lead to considerable downtime. Trained photocathode users can consistently heat clean crystals without incident, but the training can take many months.

The usefulness of a practiced eye in evaluating heating temperature led us to consider ways how this could be done quantitatively using an inexpensive IR or regular camera and correlating this measurement to crystal surface temperature. In principle, one could measure the spectral distribution of a blackbody radiator using a spectrometer, but this is inefficient and expensive. The method presented here is based on commercially available, low-cost cameras.

\section{THEORY}

Some commercially available "wavemeters" (e.g., the NewportModel\#OMM6810B) use two detectors with different spectral sensitivities. By taking the ratio of the two detector signals, these devices give a measurement of the incident light wavelength. The method we developed that is discussed in this report operates under similar principles. The wavelength distribution of light emitted by a hot body is related to its temperature. This temperature can, in turn, be determined from the signals from two different detectors as described above. Instead of two discrete detectors, our method uses a detector array (a camera CCD) that has the major advantage that it also provides a way to evaluate spatial temperature variations across a sample. As it applies to GaAs, such a device could be empirically calibrated by determining the value of the detector signal ratio at the frosting temperature, and then used to monitor subsequent photocathodes as they approached the congruent evaporation temperature during activation.

Consider a blackbody radiator at some temperature $\mathrm{T}$. The spectral radiance of this body, describing the power emitted per unit area, per unit solid angle, and per unit wavelength $\lambda$ is given by the Planck distribution

$$
B(\lambda, T)=2 h c^{2} \lambda^{-5}\left(e^{\frac{h c}{\lambda k T}}-1\right)^{-1},
$$

with $k$ the Boltzmann constant, $c$ the speed of light, and $h$ the Planck constant. ${ }^{15}$ The integrated response of a camera/filter combination to this radiation will depend on the solid angle subtended by the instrument and its wavelength-dependent overall detector sensitivity (DS). Let $g(\lambda)$ be the optical filter's transmission function and $\operatorname{DS}(\lambda)$ be the detector sensitivity of the relevant $\mathrm{CCD}$ detector, in conjunction with the transmission of any relevant internal optical element of the camera. Then the intensity registered by the instrument, per unit solid angle, is

$$
I(T)=\int B(\lambda, T) R(\lambda) d \lambda,
$$

where

$$
R(\lambda)=D S(\lambda) g(\lambda)
$$

Our method used to measure temperature relies on measuring the detected signal, $I(T)$, using two different optical filters and thus two different effective instrument response functions, $R_{1}(\lambda)$ and $R_{2}(\lambda)$. A ratio of the corresponding intensities $I_{1}(T) / I_{2}(T)$ is related to the temperature of the radiator. This formalism eliminates the dependence on the solid angle and absolute calibration of the camera/filter detector.

Early attempts at measuring color for this application used a standard "visible spectrum" camera, such as a commercial digital single-lens reflex (DSLR) camera or black and white pin hole camera. ${ }^{16-18}$ Since "color" is the desired measurement, the pixel responses are converted to the industry-accepted HueSaturation-Luminance (HSL) color space. ${ }^{19}$ The coordinate value "hue" conveys color information and, since it relies on ratios of intensities, is intensity independent. The hue is analogous to the ratio of intensities used in the wavemeter 
example just discussed. The standard HSL color space places the "hue angle" corresponding to red at $0^{\circ}$, green at $120^{\circ}$, and blue at $240^{\circ} .{ }^{20}$ Hue angle is easily calculated using the R, G, and $\mathrm{B}$ tri-stimulus intensities. ${ }^{21}$ The term hue angle is used to emphasize the analogy with traditional image processing techniques. Since the blue response is negligible at the colortemperatures required for heat cleaning GaAs crystals, we introduce a slightly modified and simpler hue angle,

$$
\theta_{\text {Hue }}=\arctan \left(\frac{I_{G}(T)}{I_{R}(T)}\right),
$$

where $I_{G}(T)$ is the camera's integrated response in the green channel (peak DS at $525 \mathrm{~nm}$ ) and $I_{R}(T)$ the response of the red channel (peak DS at $600 \mathrm{~nm}$ ).

Unfortunately, the use of commercially available cameras intended for "visible spectrum" photography causes significant problems for our application. At the temperature at which incipient local evaporation occurs (i.e., the crystal begins to frost), it is difficult to set the camera sensitivity (ISO, shutter speed, etc.) so that the green channel of the camera is statistically non-zero before the red channel saturates. In other words, cameras and subsequent display technology cannot faithfully reproduce the full range of human color vision. This is especially true for deep red and near infrared "colors." Thus, where an experienced photocathode user can perceive differences in color, the camera may not. Moreover, the red, green, and blue (RGB) image produced by the camera is intended for playback to the human observer. There is thus a significant post processing so that displayed images mimic those seen by the human eye. Accordingly, the RGB response in a recorded image will not necessarily be linear with input intensity.

Instead of detecting visible red and green wavelengths, a camera whose pixel sensitivity extends well into the near infrared should be used. Choosing a device with significant DS in the near infrared will remove the problem of simultaneously over-saturating one response while under-stimulating the second. A commercially available camera developed for the Raspberry $\mathrm{Pi}$ computer ${ }^{22}$ is available with the infrared filter removed. This device is described in greater detail below. Briefly, the three sets of pixels in this camera have peak detector sensitivity in the red, green, and blue (RGB) as usual, but also have nearly identical responses in the near infrared. Since their detector sensitivities are the same in the infrared, two images are now acquired with the camera viewing the incandescent crystal through two different filters: one with a pass wavelength of $900 \mathrm{~nm}$ and another at $800 \mathrm{~nm}$. Each filter has a full-width half-maximum transmission of $40 \mathrm{~nm}^{23,24} \mathrm{~A}$ hue angle is now calculated using Eq. (4), but replacing the indices "G" and "R" with $800 \mathrm{~nm}$ and $900 \mathrm{~nm}$. Note that this angle will have little relevance to the color perceived by a human observer.

To estimate the expected hue angle for an optimally heated crystal, consider a blackbody radiator at $650^{\circ} \mathrm{C}$. The two optical filter transmission functions of the detector, $g_{i}(\lambda)$, are modeled as Gaussian functions with center wavelengths of $900 \mathrm{~nm}$ and $800 \mathrm{~nm}$ for $g_{900 \mathrm{~nm}}(\lambda)$ and $g_{800 \mathrm{~nm}}(\lambda)$ respectively, and a full-width half-maximum of $40 \mathrm{~nm}$ for each. The spectral response DS of the Raspberry Pi camera is not documented in the manufacturer's datasheet. However, it is useful to estimate

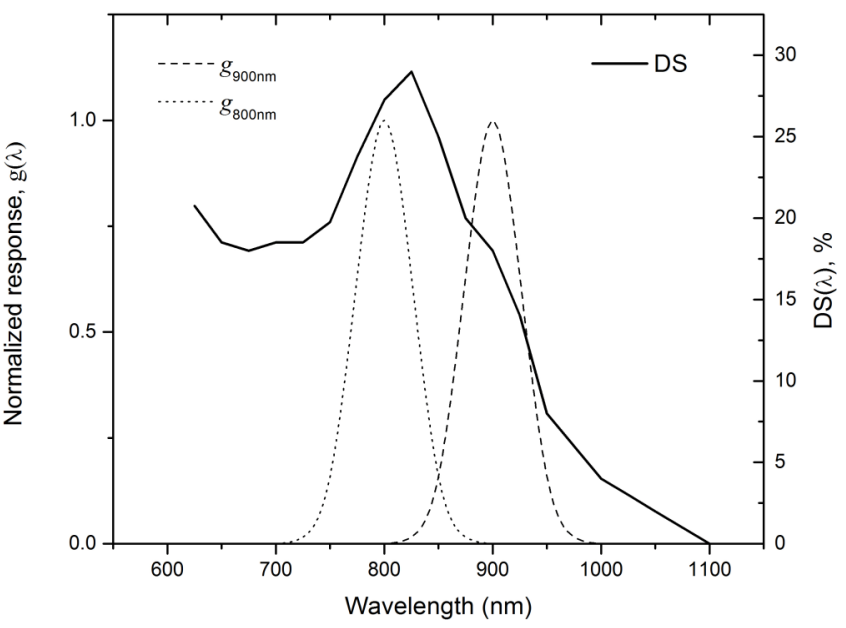

FIG. 2. Detector sensitivity, DS $(\lambda)$, for the Aptina AR0130 and two different Gaussian response functions, $g_{900 \mathrm{~nm}}(\lambda)$ and $g_{800 \mathrm{~nm}}(\lambda)$, centered at $900 \mathrm{~nm}$ and $800 \mathrm{~nm}$ respectively, and having a FWHM of $40 \mathrm{~nm}$.

the expected hue angle based on the $\operatorname{DS}(\lambda)$ of a related camera, the Aptina AR0130, which also has sensitivity in the near infrared. The DS $(\lambda)$ curve published in the Aptina AR0130 datasheet and the optical filter $g_{i}(\lambda)$ are shown in Figure 2. The effective instrument response functions, $R_{900 \mathrm{~nm}}(\lambda)$ and $R_{800 \mathrm{~nm}}(\lambda)$, for this model sensor along with the spectral power distribution, $\mathrm{B}(\lambda)$, for a $650^{\circ} \mathrm{C}$ blackbody radiator are given in Figure 3.

It is easily seen that the integrated intensity recorded for each detector will be different. Multiplying by the Planck distribution and integrating over each effective instrument response, the expected hue angle,

$$
\theta_{T=650^{\circ} \mathrm{C}}=\arctan \left(\frac{I_{800 \mathrm{~nm}}\left(T=650^{\circ} \mathrm{C}\right)}{I_{900 \mathrm{~nm}}\left(T=650^{\circ} \mathrm{C}\right)}\right),
$$

is $19.8^{\circ}$. By repeating this integration with different temperatures, it is shown that the hue angle depends monotonically on the temperature of the blackbody radiator; see Figure 4.

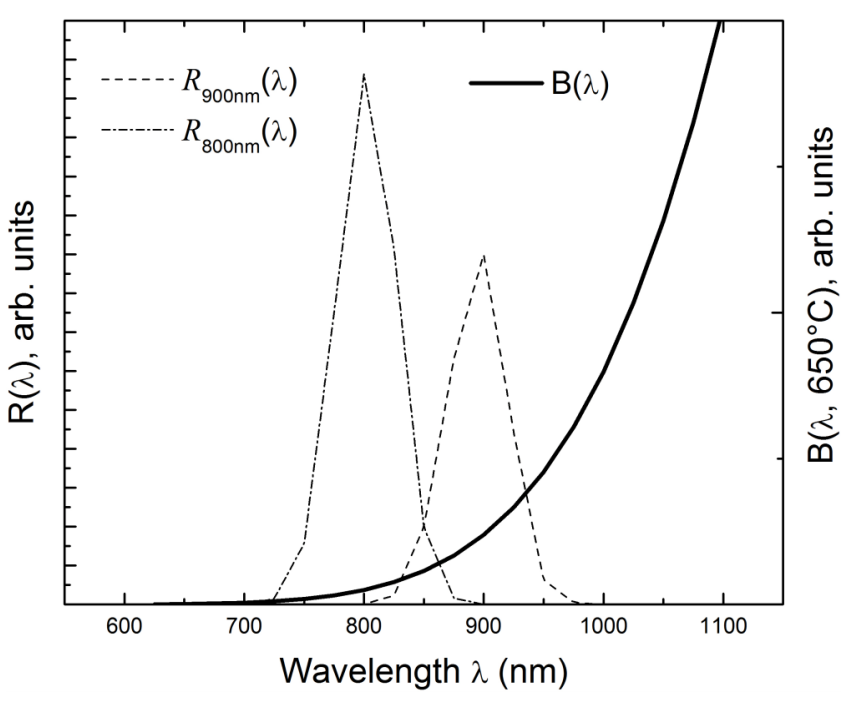

FIG. 3. The spectral radiance distribution, $B\left(\lambda, 650^{\circ} \mathrm{C}\right)$, for a model blackbody radiator at $650^{\circ} \mathrm{C}$ (solid curve) with two different effective instrument response functions, $R_{900 \mathrm{~nm}}(\lambda)$ and $R_{800 \mathrm{~nm}}(\lambda)$, obtained by multiplying $R_{i}(\lambda)$ and DS $(\lambda)$ from Fig. 2. 


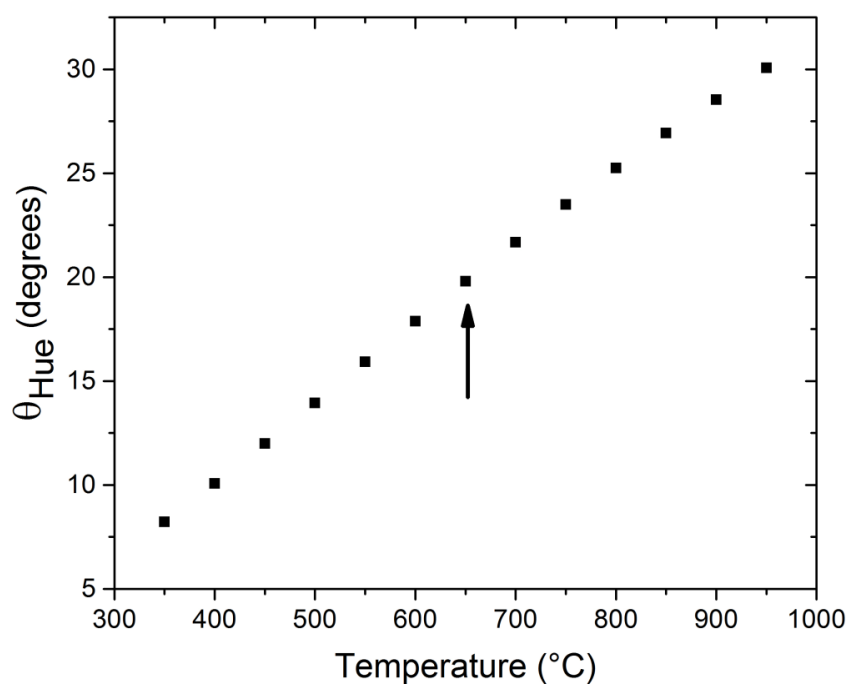

FIG. 4. Expected $\theta_{\text {Hue }}$, using the described model camera, as a function of temperature for a blackbody radiator. Using reasonable instrument responses for the two channels, this angle depends monotonically on the temperature of the observed blackbody radiator. The hue angle for an appropriate heating temperature, $650{ }^{\circ} \mathrm{C}$, is indicated. The value of $\theta_{\text {Hue }}(\mathrm{T})$ is related to the ratio of the areas beneath each effective instrument response function curve, $R_{i}(\lambda)$, for each spectral radiance distribution, $B(\lambda, T)$.

The accuracy of our measurements is limited by the bandwidths of our filters. If they are too narrow, then insufficient light is measured; if too broad, their increasing degeneracy over the wavelengths of interest reduces the measurement contrast and hence the sensitivity. Any non-linearities of the pixel response with respect to incident intensity could also have a systematic effect on our results, but we have seen no evidence of this.

\section{EXPERIMENTAL APPARATUS AND PROCEDURE}

\section{A. Color thermometer}

The temperature sensing apparatus has been constructed using a Raspberry Pi Model B, Revision 2 computer. $^{25}$ The Raspberry Pi (Raspi) is an inexpensive single-board computer that typically runs a Linux-based operating system. This platform has a significant amount of open-source software and interface hardware available. The Raspi was originally developed as an educational tool to promote the teaching of computer science and technology, but it has seen extensive use in the hobbyist community and in instructional laboratories, given its low price and versatility.

Our apparatus also uses the camera developed specifically for the Raspi which is connected through a dedicated hardware interface. There are two versions of this camera available. The standard one is based on the OmniVision OV5647 CMOS sensor and has an infrared (IR) filter installed, as do most other solid state cameras. This is necessary since the sensors are generally sensitive in the IR and would yield incorrect colors in the final output photograph if the filter were not in place. The Raspberry Pi camera can also be ordered without the IR filter-the "NoIR" option. In this camera, which we use in our apparatus, the RGB pixels are sensitive to red, green, and blue, as well as infrared.

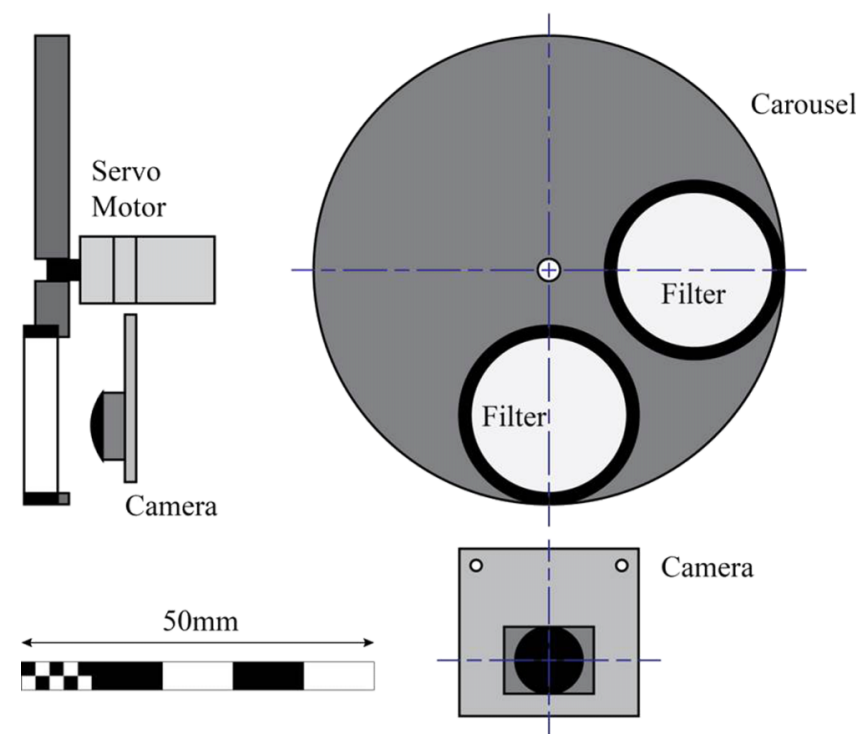

FIG. 5. Scale schematic of the camera and filter carousel assembly.

The camera is mounted behind a carousel which houses two interference filters with transmission center-wavelengths of $800 \mathrm{~nm}$ and $900 \mathrm{~nm}$ - Thorlabs part numbers FB800-40 and FB900-40, respectively (Fig. 5). The carousel is operated by a servo-motor, which is controlled through the Raspberry Pi's General Purpose Input/Output (GPIO) port. Using appropriate computer software, the computer can automatically and precisely place the appropriate filter in front of the camera. Finally, there is an enclosure constructed from $3 \mathrm{~mm}$ thick black foam-board around the camera to ensure that it does not detect stray light reflecting from the back side of the filters. Making a temperature measurement requires recording one photograph with each filter in quick succession as controlled by a computer program.

The Raspi camera is operated through the command-line Linux environment using the open-source software "Raspistill." This software allows the camera to be configured for fully manual exposure. Thus, settings such as shutter-speed and equivalent ISO are selectable. This camera can also be configured to output the raw pixel values as well as the usual post-processed image in JPG format. During this work, it was determined that the JPG images, which are intended for the human observer, did not correspond to a linear response. Instead, the camera's raw data, which represents the intensity of radiation falling on a given pixel, was used. Raw pixel values are subject to background such as noise due to surrounding read-out electronics and dark current. These offsets can be controlled through proper background subtraction. As will be explained below, the algorithm used in this work ignores the JPG image and exclusively uses the raw pixel values.

Two significantly different photocathode vacuum chamber apparatuses were used in this study to evaluate the robustness and reproducibility of our temperature monitoring methods. Each had a different mount and heating mechanism for its GaAs crystal. Thus, each system had unique thermal losses due to heat conduction and radiation. The same Raspi camera was used for both setups. 
GaAs photocathodes were cut from a GaAs wafer ( $p$-type, Zn doped, $\langle 100\rangle$, carrier concentration $\left.\sim 2 \times 10^{-19} \mathrm{~cm}^{-3}\right){ }^{26}$ Both polished and unpolished crystals were used in this study. The former had a mirror-like finish, the latter a dull matte appearance. We were initially concerned that the relationship between hue angle and temperature might depend on the surface finish, but our experiments showed this not to be the case.

Depending on the apparatus, the crystals were mounted using either a tantalum spring-clip system or binding clamps cushioned with aluminum foil. Both methods ensured that the fixtures holding the crystal established good electrical contact with it (Fig. 1). Heating of the crystal was accomplished by passing a controlled alternating current or direct current through it. Both systems had a vacuum window which allowed for observation of the photocathode at normal incidence. The nominal chamber pressures were $5 \times 10^{-8}$ Torr.

\section{B. Image processing}

The OmniVision OV5647 has a sensor resolution of $2592 \times 1944$ pixels and is organized in a Bayer matrix. ${ }^{27}$ This level of resolution is not needed in this application, so we developed software to read each $8 \times 8$ matrix of raw pixel data into one "cell" (see Figure 6). Each cell represents 16 red sensors, 16 blue sensors, and 32 green sensors. The software calculates a simple average and standard deviation of the mean $(\mathrm{SDM})$ of each $8 \times 8$ matrix of pixels and writes the information to a spreadsheet file. The error of the subsequently calculated hue angle is obtained using the SDM and standard error propagation. All RGB pixels in the camera are approximately equally sensitive in the infrared. Even if there are subtle differences in response, each cell uses the same number of red, green, or blue pixels, so each cell has the same red, green, or blue weighting in the average.

After the software reads the full frame CCD pixel data, a $324 \times 243$ array of cells is available, representing the image seen by the camera. Since the OmniVision OV5647 uses a 10-bit A-to-D converter, a pixel will saturate at a maximum value of 1024. A representative JPG image is shown in Figure 7, recorded when the camera was observing an incandescent crystal at $\sim 650^{\circ} \mathrm{C}$ through the $900 \mathrm{~nm}$ filter. The corresponding $800 \mathrm{~nm}$ JPG image is similar, but not as "bright." Example intensity information extracted from the raw data of the three regions indicated in Figure 7 is shown in Figure 8. These data, after performing a background subtraction, are used to calculate hue angle across the area of the crystal, which is also shown in Figure 8. Also visible on the right-hand side of the image (Fig. 7) are reflections from the mounting hardware. In this image, heating current generally flows along the long side of the crystal. Note also that the crystal is cracked near the lower, right-hand corner. This results in a cooler area on the crystal on the right side of the crack, and a hotter area just above since the current density is much greater in this region.

The temporal linearity of the CCD raw response is verified by recording the average intensity of the brightest region, Region 1 in Figure 7, as a function of camera "shutter-speed." Each region in Figure 7 represents an array of $7 \times 7$ cells $(56 \times 56$ pixels). Although the term "shutter speed" is used, this variable actually refers to the exposure dwell time. (This term is the common vernacular in photography and is used by the "Raspistill" software.) Figure 9 shows two different sets of background-corrected data for Region 1 corresponding to $900 \mathrm{~nm}$ and $800 \mathrm{~nm}$ intensities with two different heating powers. From this graph, one can conclude that the detector's

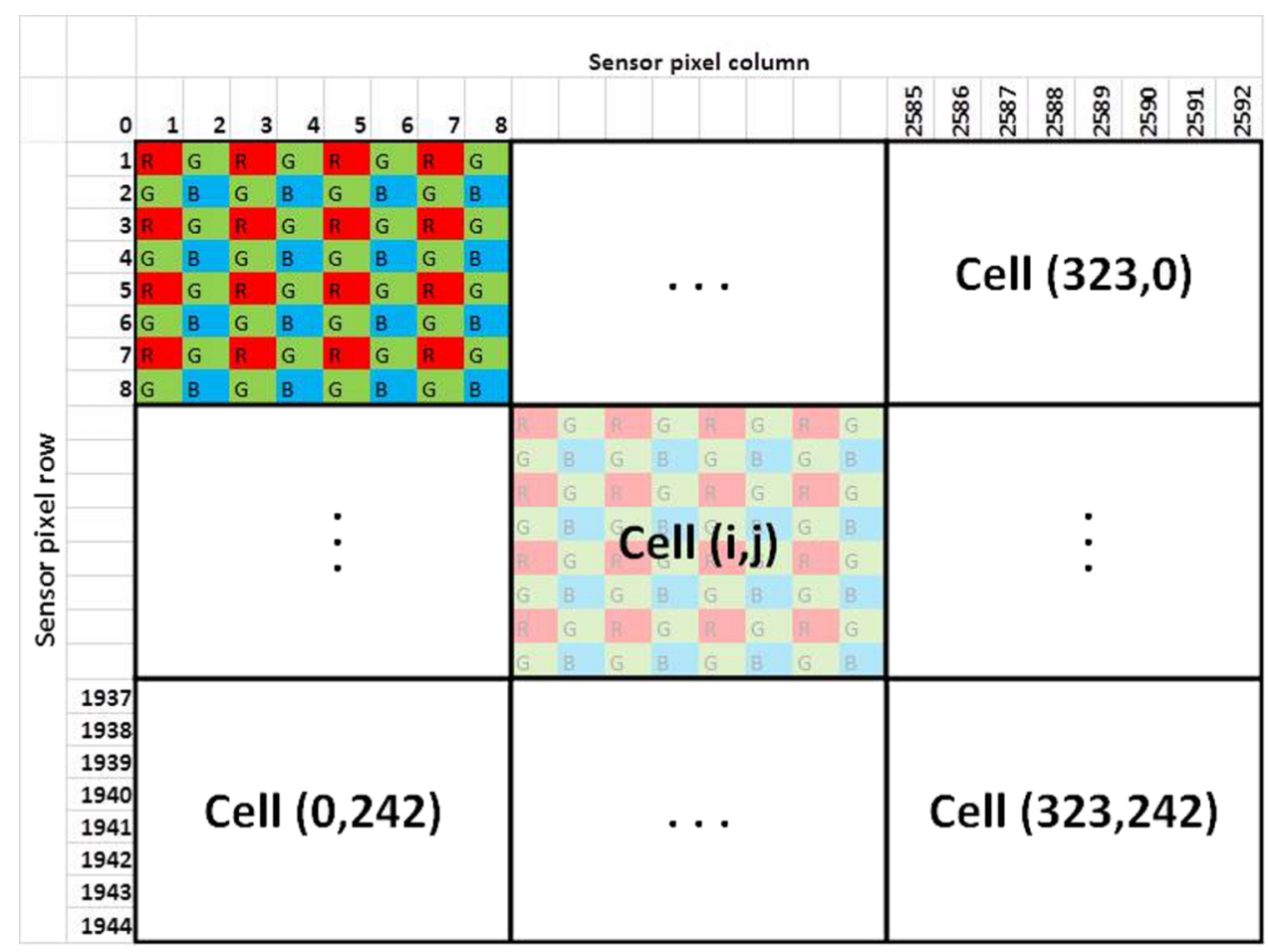

FIG. 6. Computer software developed for this project reads raw CCD Bayer pixel information from the Raspi camera in $8 \times 8$ blocks of pixels and averages their values into a single "cell" (see text). 


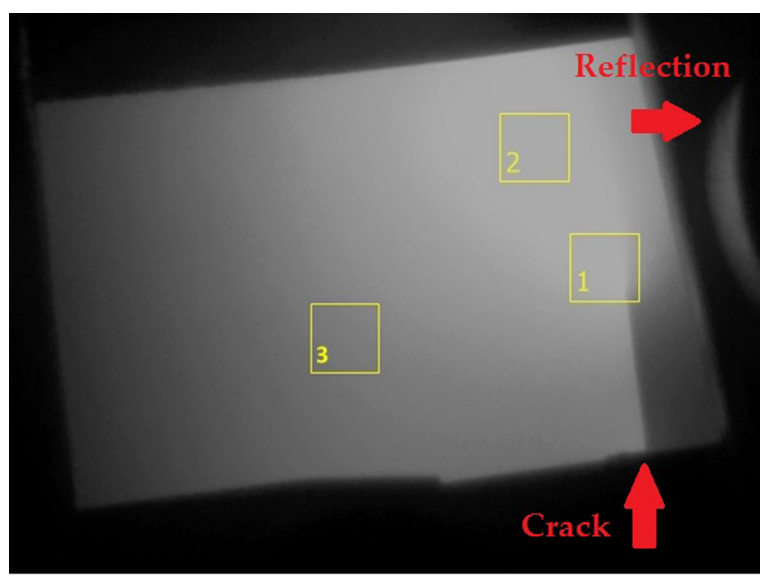

FIG. 7. Representative $600 \times 448$ pixel image of an incandescent GaAs crystal taken with the Raspi camera through the $900 \mathrm{~nm}$ filter. The corresponding $800 \mathrm{~nm}$ image is similar. The crystal is in thermal equilibrium at $\sim 650^{\circ} \mathrm{C}$, heated with $8.9 \mathrm{~W}$ of electrical power. Reflections from mounting hardware are visible on the right side of the image. Note that the crystal is cracked near the lower right edge. The raw CCD data from this image are used to generate the information in Figure 8. The indicated regions are used later in the text.

response is linear with exposure time (shutter-speed). Thus the hue angle will be independent of exposure time provided that the sensors are not saturated or statistically zero after background is subtracted. Second, it is apparent that the intensity of radiation from the hottest region is greater at greater heating powers as expected.

Figure 10 shows the weighted average of $\theta_{\text {Hue }}$ for each of the three regions shown in Figure 7 as a function of heating power. Looking primarily at the data for Regions 2 and 3, we see that when the heating power is small, the temperature is approximately constant across the face of the crystal. The drop in $\theta_{\text {Hue }}$ from 4 to $4.5 \mathrm{~W}$ in Region 1 may be due to enhanced thermal conduction between the GaAs crystal and its mounting hardware caused by the thermal expansion of both. This effect may be enhanced by the crack near Region 1 .

\section{RESULTS}

Two different approaches to determine empirically the hue angle corresponding to the optimum temperature for heat cleaning have been considered. In Method I, the crystal is heated until it is at the correct temperature as judged by an expert photocathode user. Then NIR photos are acquired, and the hue angle for the hottest region determined. Method 2 attempts to remove the subjectivity of the human observer by iteratively heating the crystal until it becomes frosted. The temperature at which crystal frosting occurs is then determined by analysis of the NIR photos.

\section{A. Method 1}

For Method 1, the crystal was resistively heated using an AC current source. In general, GaAs photocathodes are heated via one of two heating procedures: a "fast-hot" procedure or a "slow-cool" procedure. Both procedures are effective. The fast-hot method gradually heats the crystal a)

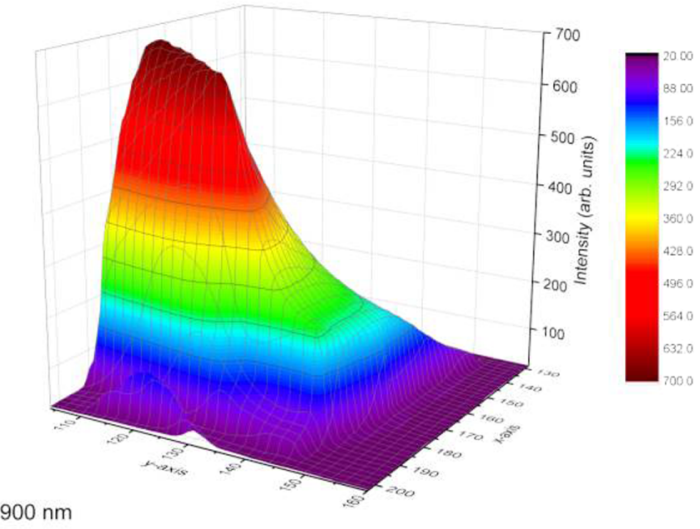

b)

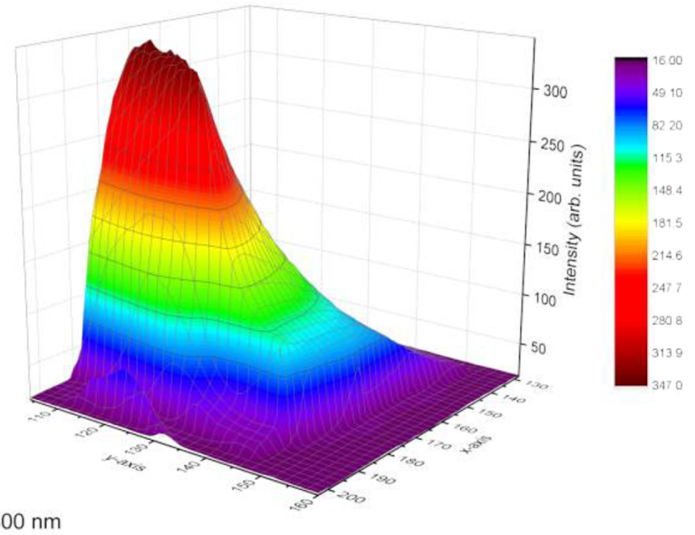

c)

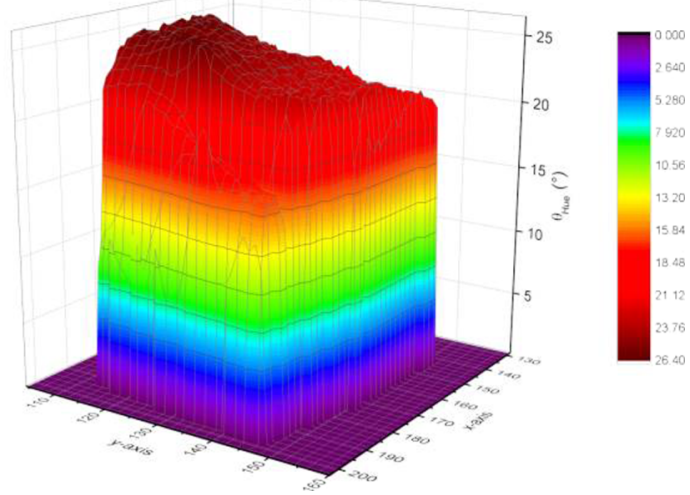

FIG. 8. Example $75 \times 56$ array of cells extracted from the images like those shown in Figure 7. Shown are the corresponding $900 \mathrm{~nm}$ (a) and $800 \mathrm{~nm}$ (b) data taken from one zone measurement. These data are used to calculate the hue angle and generate a Hue angle map (c) for the crystal, zone 2, shown in Figure 7.

to between 610 and $650{ }^{\circ} \mathrm{C}$ and holds that temperature for 10-20 min. This procedure is quicker but has a greater risk of frosting the crystal. The slow-cool method heats the crystal to 590-610 ${ }^{\circ} \mathrm{C}$ and holds that temperature for one to two $\mathrm{h} .{ }^{12}$ The current is raised slowly, at about 0.5 A per $10 \mathrm{~min}$, until the desired final temperature is reached. Typically this occurred at $\sim 2 \mathrm{~A}$ for our samples. This technique is less reliable at producing quality photocathodes, but also has less risk of frosting the crystal. While our group has had greater success with the fast-hot method, other labs often use a version of the slowcool method for both their bulk GaAs crystals and their high polarization, strained superlattice cathodes. ${ }^{28}$ 


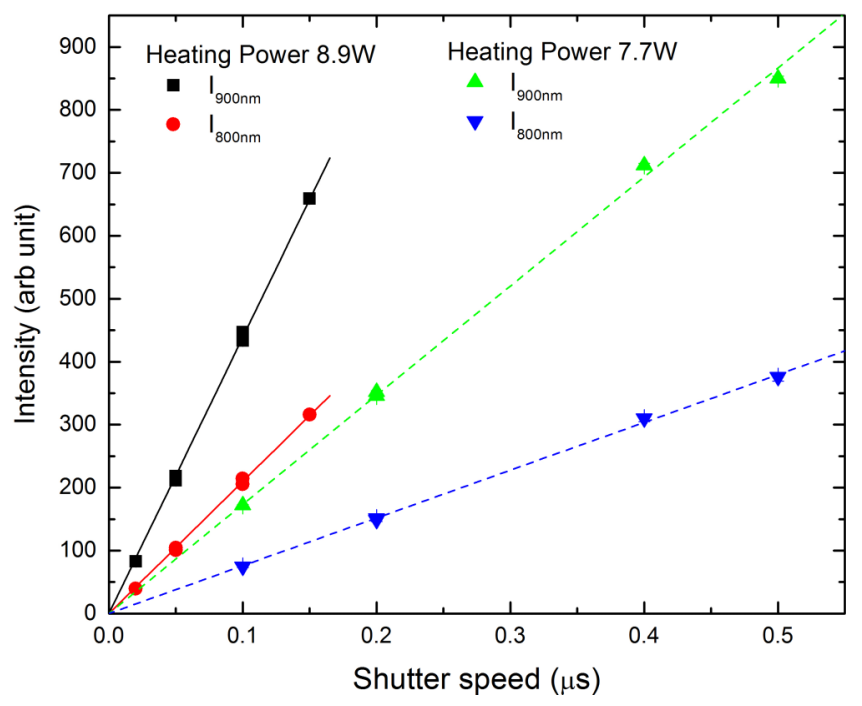

FIG. 9. Background-corrected intensity of Region 1 (Figure 7) as a function of camera shutter speed. Shown are two sets of data for two different heating powers of the same crystal. The lines are least-squares linear fits to the data forced through the origin. The data point error bars are smaller than the data points.

In the Method 1 case, an experienced photocathode user visually monitored the crystal until it reached the minimum desirable temperature for a fast-hot heat clean, and determined the hue angle. The power was increased and the hue angle recorded again until the user deemed it unwise to increase the heating power at the risk of frosting the crystal. These results are shown in Figure 11 for a variety of independent trials in which the crystal started at room temperature each time. The weighted average $\theta_{\text {Hue }}$ of these trials was $22.9(2)^{\circ}$. This value represents the hue angle appropriate for producing $\mathrm{GaAs}$ photocathodes using the fast-hot method. The hue temperature determined by Method 1 can serve as the standardized benchmark for heat cleaning. As such, it is an excellent tool for training new photocathode users.

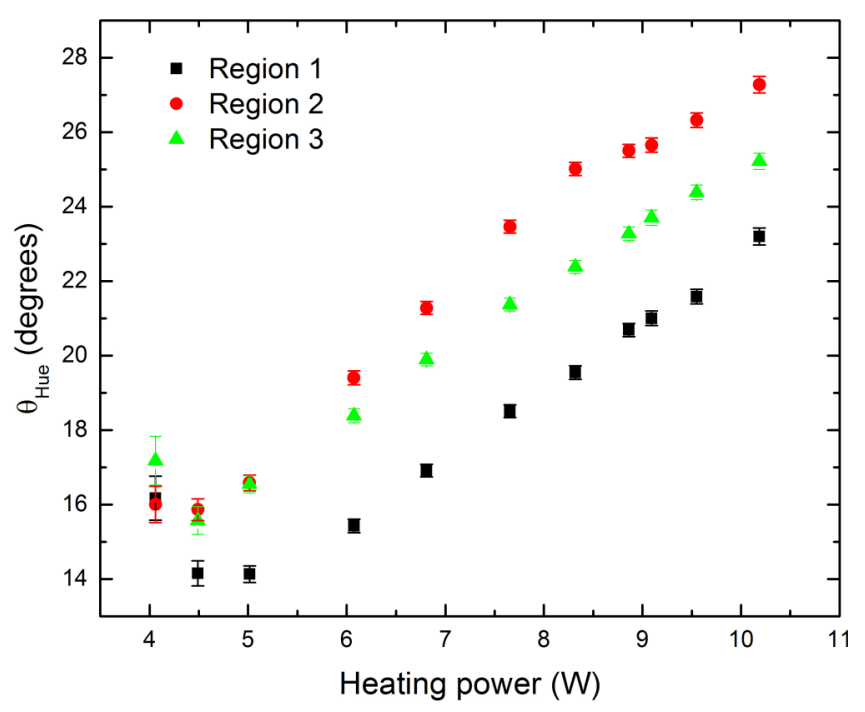

FIG. 10. Hue temperature as a function of crystal heating power for the regions indicated in Figure 7.

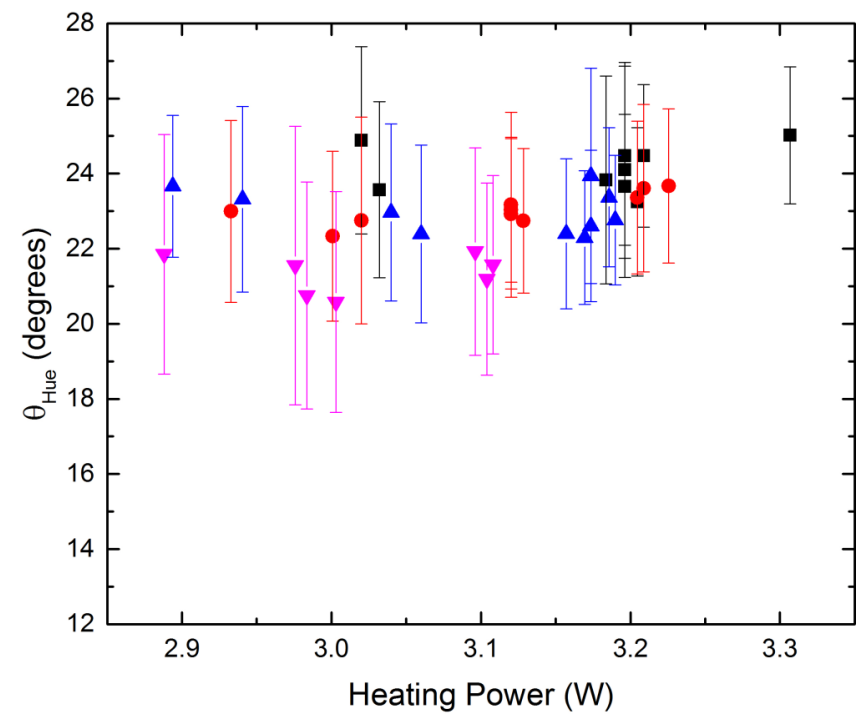

FIG. 11. The measured hue angle of the hottest section of a heated GaAs crystal as a function of heating power for various trials denoted by the different symbols. The left- and right-most points corresponding to each trial bracket a temperature range in which photocathodes can be produced without unnecessarily risking frosting the crystal. A similar hue angle is routinely reached during heating, suggesting the robustness of this technique. Individual uncertainties correspond to the SDM for pixel responses from a $7 \times 7$ cell region.

\section{B. Method 2}

In the second method, the crystal heating was cycled from zero to some maximum power and data acquired for the sample's hottest region. For each heating cycle, the final heating power was incrementally raised. After each heating cycle, the heating power was removed so that the crystal state could be checked, and the process repeated with a higher final power. This process was conducted on two different crystals using the same chamber. After the first crystal frosted, it was replaced and the experiment was conducted again.

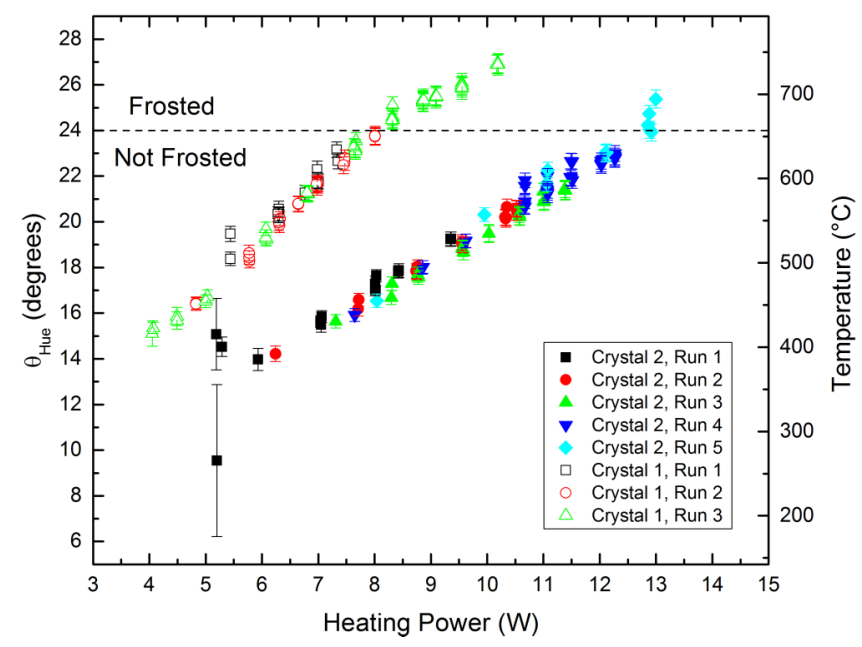

FIG. 12. Hue angle of the hottest portion of the heated GaAs crystal as a function of heating power for two different crystals. Above $\theta_{\mathrm{Hue}}=24^{\circ}$ the crystals begin to frost. The temperature scale was not determined experimentally, but instead set such that $\theta_{\text {Hue }}=24^{\circ}$ corresponds to the congruent evaporation temperature. Below this temperature, $\theta_{\text {Hue }}$ is roughly proportional to the crystal temperature (see Fig. 4). 
As illustrated in Figure 12, the hue angle for the hottest portion of the crystal just prior to frosting is reproducible. Changing the crystal means that there will be different thermal conduction characteristics due to variability in the mounting hardware, so it is not surprising that the hue-power curve is different for different crystals. Also shown in Figure 12 is the frosting "state" of the crystal at the end of a given heating cycle. Based on this information, the crystal begins to frost at $\theta_{\text {Hue }}=24.0(5)^{\circ}$. As expected, $\theta_{\text {Hue, Method } 1}<\theta_{\text {Hue, Method } 2}$ as Method 1 measures a conservative temperature to heat clean a photocathode, and Method 2 measures the temperature at which the photocathodes actually become frosted.

\section{CONCLUSIONS}

An inexpensive system to provide in situ temperature measurements of a GaAs crystal (or anything else for that matter) has been developed. It is based on comparing pixel responses from an inexpensive camera at different infrared wavelengths. Using a simple model, in which the wavelengthdependent response of the instrument was approximated, a theoretical color temperature hue angle value of $19.8^{\circ}$ was expected at the onset of heating. An experienced photocathode user (Method 1) determined the proper angle to be $22.9(2)^{\circ}$. It was also determined empirically (Method 2) that the hue angle above which frosting occurs is $24.0(5)^{\circ}$.

Because the NIR camera is more sensitive to the light emitted by the photocathode, the onset of the temperature regime that produces good photocathodes is more easily distinguished by a NIR camera than the human eye. Owing to the use of NIR cameras in our laboratory we have, to date, never frosted a crystal due to improper heating.

By adding heating power control from the computer, this system can be easily modified to provide full automatic heat cleaning of a GaAs crystal. Looking forward, the approach presented here also provides a means to produce temperature maps that users can compare and correlate with quantum efficiency maps. ${ }^{29,30}$

\section{ACKNOWLEDGMENTS}

This work was funded by the National Science Foundation through Grant No. PHY-1505794. The authors gratefully acknowledge the assistance of J. M. Dreiling and E. Brunkow. The craftsmanship of the University of Nebraska-
Lincoln Physics and Chemistry instrument shop was essential to this work. The authors wish to thank the two referees of this paper who provided comments that significantly improved the manuscript.

${ }^{1}$ D. T. Pierce and F. Meier, Phys. Rev. B 13, 5484 (1976).

${ }^{2}$ F. C. Tang, M. S. Lubell, K. Rubin, A. Vasilakis, M. Eminyan, and J. Slevin, Rev. Sci. Instrum. 57, 3004 (1986).

${ }^{3}$ C. K. Sinclair, P. A. Adderley, B. M. Dunham, J. C. Hansknecht, P. Hartmann, M. Poelker, J. S. Price, P. M. Rutt, W. J. Schneider, and M. Steigerwald, Phys. Rev. ST Accel. Beams 10, 023501 (2007).

${ }^{4}$ D. A. Orlov, C. Krantz, A. Wolf, A. S. Jaroshevich, S. N. Kosolobov, H. E. Scheibler, and A. S. Terekhov, J. Appl. Phys. 106, 054907 (2009).

${ }^{5}$ C. T. Foxon, J. A. Harvey, and B. A. Joyce, J. Phys. Chem. Solids 34, 1693 (1973).

${ }^{6}$ B. Goldstein, D. J. Szostak, and V. S. Ban, Surf. Sci. 57, 733 (1976).

${ }^{7}$ D. T. Pierce, R. J. Celotta, G.-C. Wang, W. N. Unertl, A. Galejs, C. E. Kuyatt, and S. R. Mielczarek, Rev. Sci. Instrum. 51, 478 (1980).

${ }^{8}$ K. Wijayaratna, Ph.D. dissertation (University of Missouri-Rolla, 1992).

${ }^{9} \mathrm{~K}$. W. Trantham, Ph.D. dissertation (University of Nebraska-Lincoln, 1996).

${ }^{10}$ A. S. Green, Ph.D. dissertation (University of Nebraska-Lincoln, 2003).

${ }^{11}$ J. W. Maseberg, Ph.D. dissertation (University of Nebraska-Lincoln, 2009).

${ }^{12}$ J. M. Dreiling, Ph. D. dissertation (University of Nebraska-Lincoln, 2014).

${ }^{13}$ J. Grames, Thomas Jefferson National Lab Technical Note TN-04-0055, 2004.

${ }^{14}$ P. J. Timans, J. Appl. Phys. 117, 205901 (2015).

${ }^{15}$ M. Planck, The Theory of Heat Radiation (Dover, New York, 1959). This publication is an English translation of the second edition of Planck's work entitled, Waermestrahlung, published in 1913.

${ }^{16}$ Canon $700 \mathrm{~d}$

${ }^{17}$ Nikon D90.

${ }^{18}$ Black and white pin hole camera: Ez Watch 15-CG30A-1.

${ }^{19} \mathrm{G}$. H. Joblove and D. Greenberg, ACM SIGGRAPH Comput. Graphics 12, 20 (1978).

${ }^{20}$ M. K. Agoston, Computer Graphics and Geometric Modeling: Implementation and Algorithms (Springer, London, 2005).

${ }^{21}$ F. Preucil, in TAGA Proceedings (Technical Assoc. of the Graphic Arts, 1953), pp. 102-110.

${ }^{22}$ Raspberry Pi NoIR Camera Board - Infrared-sensitive Camera, Raspberry Pi Foundation.

${ }^{23}$ Bandpass filter with center wavelength $(\mathrm{CWL})=800 \pm 8 \mathrm{~nm}$, bandpass FWHM $=40 \pm 8$ nm, Part \# FB800-40, Thorlabs.

${ }^{24}$ Bandpass filter CWL $=900 \pm 8 \mathrm{~nm}$, bandpass FWHM $=40 \pm 8 \mathrm{~nm}$, Part \# FB900-40, Thorlabs.

${ }^{25}$ Raspberry Pi 1 Model B revision 2 Computer, Raspberry Pi Foundation.

${ }^{26}$ Crystal Specialties International.

${ }^{27}$ OV05647 CMOS Image Sensor, OmniVision Technologies.

${ }^{28}$ M. Stutzman, Thomas Jefferson National Accelerator Facility, private communication (2012).

${ }^{29}$ M. Baylac, P. Adderley, J. Brittian, J. Clark, T. Day, J. Grames, J. Hansknecht, M. Poelker, M. Stutzman, A. T. Wu, and A. S. Terekhov, Phys. Rev. Spec. Top.-Accel. Beams 8, 123501 (2005).

${ }^{30}$ R. R. Mammei, R. Suleiman, J. Feingold, P. A. Adderley, J. Clark, S. Covert, J. Grames, J. Hansknecht, D. Machie, M. Poelker, T. Rao, J. Smedley, J. Walsh, J. L. McCarter, and M. Ruiz-Osés, Phys. Rev. Spec. Top.-Accel. Beams 16, 033401 (2013). 